\title{
AN EFFiCiEnt DyNAMic CALl AdMission CONTROL FOR 4G AND 5G NETWORKS
}

\author{
Maharazu Mamman ${ }^{1}$ and Zurina Mohd Hanapi ${ }^{2}$ \\ ${ }^{1}$ Department of Computer Science, Federal College of Education Katsina, \\ P.M.B. 2041 Katsina State, Nigeria \\ ${ }^{2}$ Department of Communication Technology and Networks, Faculty of \\ Computer Science and Information Technology, Universiti Putra Malaysia, \\ Serdang 43400, Malaysia
}

\begin{abstract}
The goal for improved wireless communication between interconnected objects in a network has been long anticipated. The present Long Term Evolution (LTE) fourth-generation (4G) network does not allow the variety of services for the future need, as the fifth-generation $(5 G)$ network is faster, efficient, reliable, and more flexible. The $5 G$ network and call admission control (CAC) are best certainty that defines the elementary principles of the smart cities of the upcoming $5 G$ network technology. It is predicted that substantial CAC in the smart cites environment where millions of wireless devices are connected, communication will be granted based on latency, speed, and cost. Furthermore, the present CAC algorithm suffers from performance deteriorates under the $4 G$ network because of the adaptive threshold value used to determine the strength of the network. In this paper, a novel CAC algorithm that uses dynamic threshold value for smart cities in the $5 G$ network to address performance deterioration is proposed. Simulation is used to evaluate the efficacy of the proposed algorithm, and results show that it significantly performs better than do other algorithm based on the metrics measured.
\end{abstract}

\section{KEYWORDS}

Long Term Evolution, 4G, 5G, Networks, Call admission control

\section{INTRODUCTION}

The communication industry commenced with Advanced Mobile Phone System (AMPS) also known as $1 \mathrm{G}$ in form of analog mode. The next advancement was the Global System for Mobile (GSM) the first digital communication method referred to as $2 \mathrm{G}$. An improvement of $2 \mathrm{G}$ in terms of data rate yield to the development of the Universal Mobile Telecommunication System (UMTS) is a 3G technology. With the improvement in data rates and high need for bandwidth Mobile Wimax and LTE (4G) evolved to overcome the limitations of 3G. Nowadays, sophisticated communication technology is $5 \mathrm{G}$ [1]. The architecture of the $4 \mathrm{G}$ network consisted of three key sub-networks via Evolved Universal Terrestrial Radio Access Network, evolved packet network, and broadband network. Similarly, the 5G networks consist of all Internet Protocol (IP) for mobile and wireless network interoperability which comprises user terminals and an autonomous radio access technology. Radio resource management is one of the key research trends in both $4 \mathrm{G}$ and $5 \mathrm{G}$. The $\mathrm{CAC}$ is one of the fundamental strategies for radio resource management.

David C. Wyld et al. (Eds): SPPR, NECO, GridCom, ICCSEA, SCAI, UBIC, SEMIT, MLDS - 2020 
Although the LTE 4G network is good, yet it has some defects associated with it, for example, its environment and set back of transmission order as in the cases of $1 \mathrm{G}, 2 \mathrm{G}$, and $3 \mathrm{G}$. Several isolated rural areas and many structures in the urban cities have network access because of the present transmission orders and equipment. This has to be improved to satisfy the predicted $5 \mathrm{G}$ network that has a variety of different skills that are proficient in providing transmission order and may other purposes [2]. The present anticipated wireless communication for 5G will help the liberated implication of several information open which a well-known CAC in the smart city relies on. The CAC in the smart city and 5G are carefully correlated because the amazing large data created by the CAC will want the flexibility that $5 \mathrm{G}$ is capable to quarter and hence CAC in the smart city will drive the advanced form of the $5 \mathrm{G}$ network.

The organization of this paper is as follows. Section 2 provides several related works. Section 3 presented the proposed algorithm and its details. Simulation results are illustrated in Section 4, while conclusions and future work are given in Section 5.

\section{RELATED WORKS}

A lot of investigations have been done on $\mathrm{CAC}$ in $4 \mathrm{G}$ and $5 \mathrm{G}$ both at academia and industries, hereafter researchers have presented many proposals towards such directions. The authors in [3] proposed the CAC algorithm for Energy Saving in 5G H-CRAN Networks intending to minimize the total power consumption in the H-CRAN using switch sleep mode strategy. However, call blocking probability (CBP) and call dropping probability (CDP) are completely ignored which are the building blocks in CAC. In [4], Fuzzy logic-based CAC in 5G cloud radio access networks with preemption was presented. The algorithm used a cloud bursting method during the congestion period to preempt delay-tolerant low-priority and outsourced penalty charges for the public cloud. It achieved a low CBP 5\% but an increase in CDP. CAC for Real-Time and Nonreal-time Traffic for Vehicular LTE Downlink Networks was proposed in [5]. The algorithm aims to accept or reject calls based on user priority. Besides, it classified calls into handoff and new calls while the traffic requests are categorized into real-time and non-real-time.

A Hybrid Approach to CAC in 5G Networks was proposed in [6] using neurofuzzy controller as one of the strategies of artificial intelligence. The algorithm increases the quality of service (QoS) by minimizing the CBP of the new incoming calls in a network. However, the CDP was significantly increased. In [7], a comprehensive survey has been presented that described the current research state-of-the-art of 5G Internet of Things (IoT), key enabling technologies, and main research trends as well as challenges in 5G IoT.

Similarly, Simulation analysis of key technology optimization of 5G mobile communication based on IoT technology was proposed in [8]. The algorithm aimed to minimize the base station energy power consumption and improve network energy efficiency to achieved good communication quality. The base station was tested based on four working loads: zero, light, normal, and heavy. In [9], Energy Efficient Proposal for IoT CAC in 5G Network was presented. The algorithm aimed to minimize energy consumption using CAC modeling for IoT in new radio access 5G networks. However, the CBP and CDP are ignored which resulted in network performance degradation.

An Efficient admission control and resource allocation mechanisms for public safety communication over 5G network slice was proposed in [10]. The authors provide an overview of how CAC and resource allocation can be deployed efficiently in the 5G network. In [11], An Adaptive CAC with Bandwidth Reservation for Downlink LTE Networks was presented. The algorithm uses an adaptive threshold value to adjust the network environment under heavy traffic load. It achieved maximum throughput for Best-effort traffic (BE), decreased CBP and CDP. 
However, the algorithm was implemented in LTE $4 \mathrm{G}$ networks. Therefore, in this paper, a Dynamic efficient CAC for both $4 \mathrm{G}$ and $5 \mathrm{G}$ networks is proposed which is an improvement of [11], therein provides better throughput for BE traffic, a significant decrease in CBP and CDP.

\section{Proposed Algorithm}

In this paper, we proposed a new CAC strategy named "A Dynamic Efficient CAC for both $4 \mathrm{G}$ and 5G networks" which is an improvement of Adaptive CAC with Bandwidth Reservation for Downlink LTE Networks. Firstly, the limitations of the Adaptive CAC algorithm are outlined. The algorithm uses an adaptive threshold hold value to achieve maximum utilization of resources. But this causes an increase of CBP and CDP which deteriorates the network performance. To eradicate the shortcoming of Adaptive CAC, the proposed algorithm uses a dynamic threshold value that can be applied in both $4 \mathrm{G}$ and $5 \mathrm{G}$ networks thus increase the effective network performance, reduce CBP and CDP. The dynamic threshold value is calculated based on Equation 1. If the threshold value is less than the total bandwidth, then handoff calls or new calls are accepted, otherwise, the calls are rejected. The $\theta$ value is set 20 to enable much traffic for handoff calls to be admitted into the network, while new calls are blocked when the number of calls is above the $\theta$ value. Figure 1 illustrates a flow diagram of how the proposed algorithm works.

$$
\text { Threshold }_{\text {Dynamic }}=\theta \times \text { Handoff_call }_{\text {prob }}+N_{\text {ew_call }} \text { prob }
$$

where $\theta$ is equal 20, Handoff_call prob $_{\text {represents }}$ the handoff call probability and $\mathrm{New}_{-} \mathrm{Call}_{\text {prob }}$ denotes the new call probability respectively.

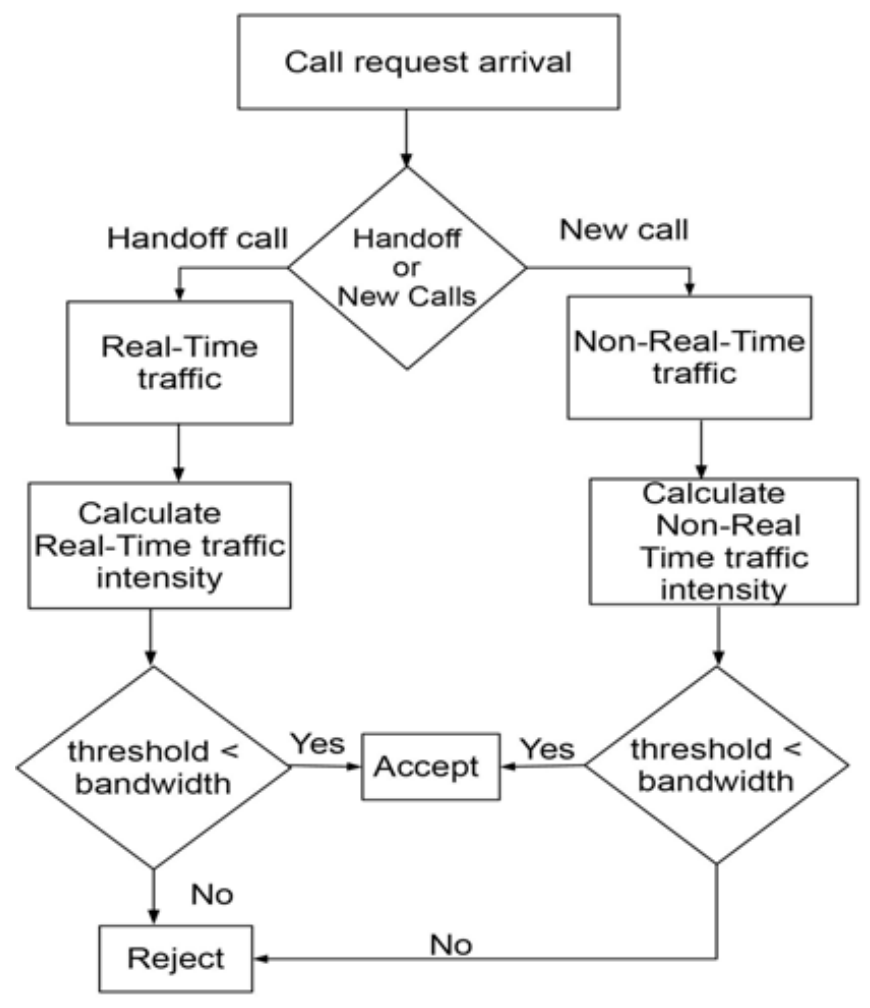

Figure 1. Proposal Algorithm 


\section{Simulation Results}

This section evaluates the performance of the proposed algorithm. The proposed algorithm is compared with the Adaptive CAC algorithm and simulation results are obtained using MATLAB system-level simulator. Three valuable metrics are used to measure the performance of the proposed algorithm which includes throughput, call blocking probability, and call dropping probability. The simulation scenario consists of one hexagonal cell with a $500 \mathrm{~m}$ radius. The total bandwidth used is $5 \mathrm{MHz}$ with 25 resource block per slot of 12 subcarriers spacing. The calls that arrived at the network environment are classified as handoff calls which includes real-time traffic that has the highest priority for instance live streaming and new call consist of non-real-time traffic which has low priority example YouTube and best-effort traffic for example email. The arrival rate for both real-time and non-real-time is Poisson distribution, while the service time is exponentially distributed. The simulation time is 500s, while an average of 20 times is used to obtain the simulation results. The simulation parameters are listed in Table 1.

Table 1. Simulation Parameters

\begin{tabular}{|l|l|}
\hline Parameter & Description \\
\hline Bandwidth & $5 \mathrm{MHZ}$ \\
\hline Number of Resource Blocks & 25 \\
\hline Total Transmission Time & $1 \mathrm{~ms}$ \\
\hline Simulation Time & $500 \mathrm{~s}$ \\
\hline Mobile Distribution & Uniform \\
\hline Traffic arrival rate & 1 \\
\hline
\end{tabular}

Figure 2 shows the throughput of the proposed algorithm against the Adaptive CAC algorithm for the BE traffic. From the figure, it can be observed that the proposed algorithm has shown significant improvement in the $5 \mathrm{G}$ environment thus, prevent starvation of Best effort traffic.

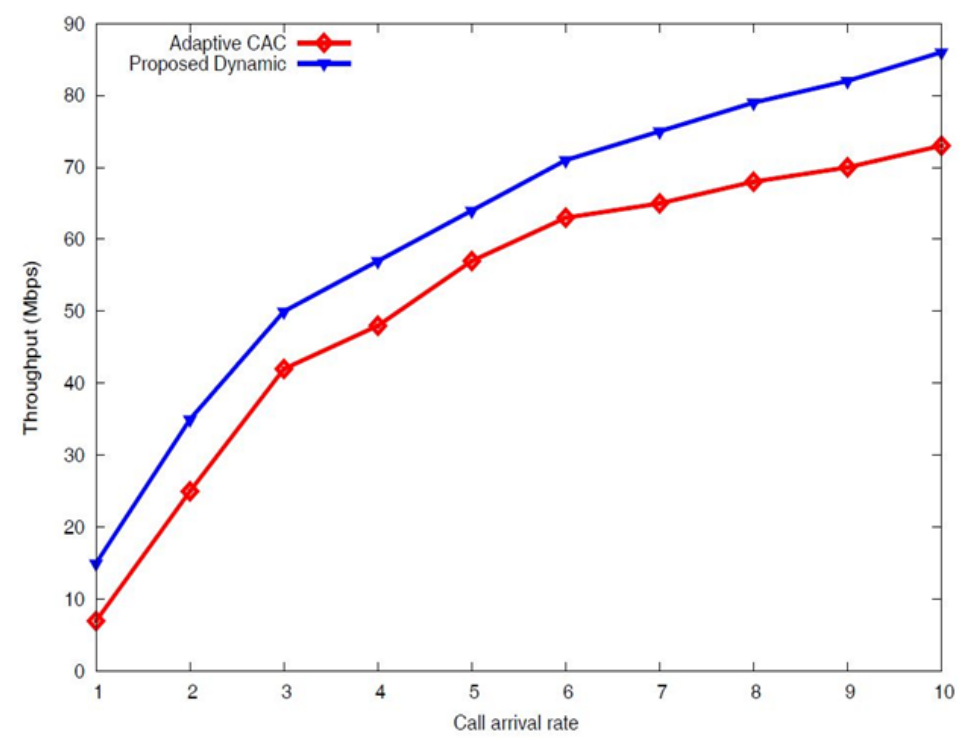

Figure 2. Throughput Best effort traffic

Figures 3 and 4 illustrate the results of CBP and CDP. The proposed algorithm has the minimum probabilities due to the dynamic use of threshold value whereby user-requested are granted based on Equation 1. Figure 3 illustrates the New call CBP for both the proposed algorithm and the Adaptive CAC algorithm. When the traffic arrival is increased the proposed algorithm performs 
better than the Adaptive CAC algorithm by decreasing the new call CBP. This was caused because of the introduction of new call criteria to prevent starvation of Best-effort traffic as well as the waste of resources of handoff calls. To this end, several new calls will be admitted into the network. Figure 4 shows the Handoff call CDP was proposed algorithm is compared with the Adaptive CAC algorithm. When the numbers of calls are increased the proposed algorithm significantly outperforms the Adaptive CAC algorithm by minimizing the new call CDP this was attributed due to the dynamic adjustment of the threshold value. Consequently, the proposed algorithm guarantees the QoS of much traffic.

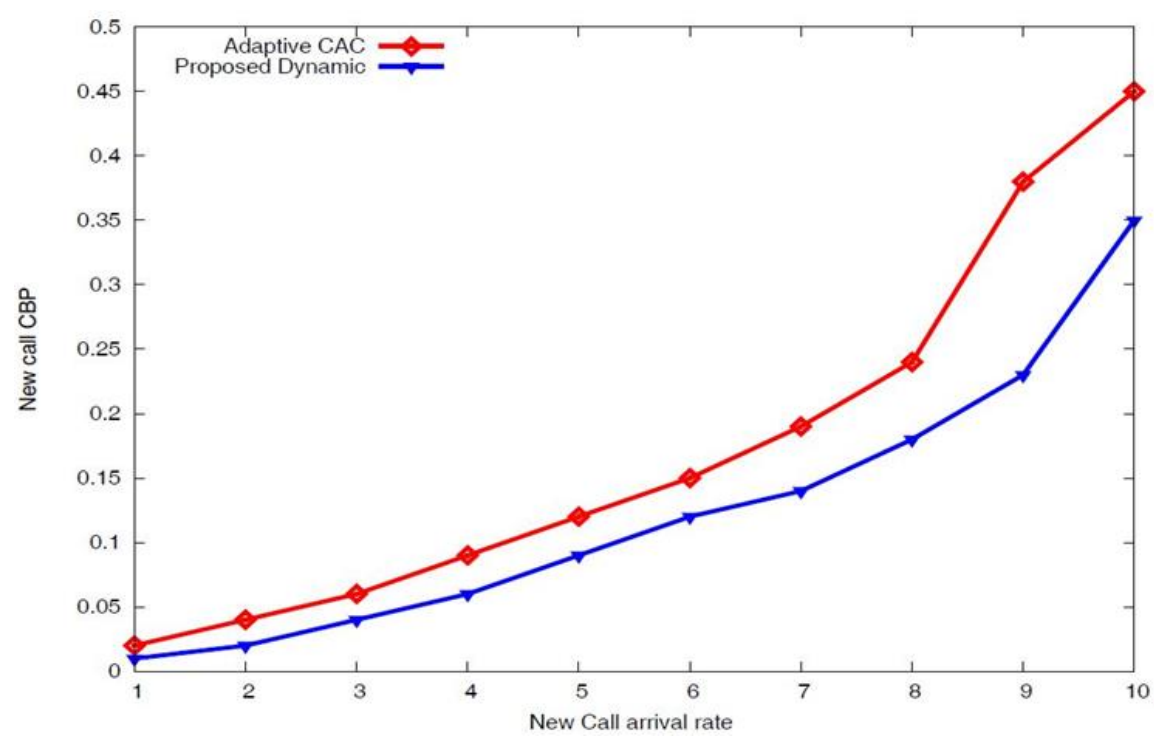

Figure 3. New call CBP

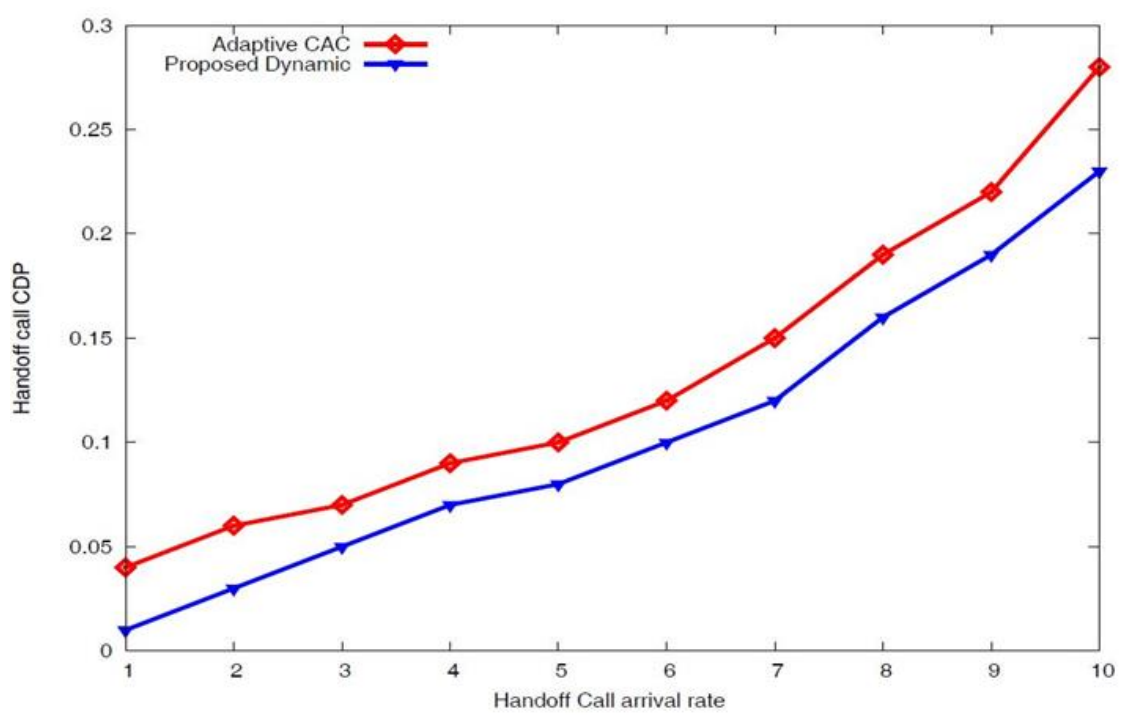

Figure 4. Handoff call CDP

\section{CONCLUSIONS ANd Future WORK}

In this paper, an Efficient Dynamic Call Admission Control for 4G and 5G Networks has been proposed to prevent starvation best-effort traffic and improve the efficient use of resources in $5 \mathrm{G}$ 
networks. The algorithm uses a dynamic threshold value to admit may mobile users to the network which enables effective use of network resources. Extensive simulation results using MATLAB system-level simulator illustrates that the proposed algorithm significantly outperformed the Adaptive CAC by minimizing the CBP, CDP, and improved throughput. This shows that the proposed algorithm is a valid candidate for $5 \mathrm{G}$ networks. In the future, we intend to test the algorithm using many load scenarios by a mathematical model.

\section{ACKNOWLEDGEMENTS}

This work is supported by Geran Putra Berimpak Universiti Putra Malaysia (9659400).

\section{REFERENCES}

[1] P. Sule and A. Joshi, "International Journal of Computer Science and Mobile Computing Architectural Shift from 4G to 5G Wireless Mobile Networks," Int. J. Comput. Sci. Mob. Comput., vol. 3, no. 9, pp. 715-721, 2014, [Online]. Available: www.ijcsmc.com.

[2] M. E. Ezema, F. A. Okoye, and A. O. Okwori, "A framework of 5G networks as the foundation for IoTs technology for improved future network,” Int. J. Phys. Sci., vol. 14, no. 10, pp. 97-107, 2019, doi: 10.5897/IJPS2018.4782.

[3] R. Gbegbe, O. Asseu, K. E. Ali, G. L. Diety, and S. Hamouda, "Call Admission Control Algorithm for Energy Saving in 5G H-CRAN Networks,” Asian J. Appl. Sci., vol. 10, no. 4, pp. 179-185, 2017, doi: 10.3923/ajaps.2017.179.185.

[4] T. Sigwele, P. Pillai, A. S. Atm, and F. Y. Hu, "Fuzzy logic-based call admission control in 5G cloud radio access networks with preemption," EURASIP J. Wirel. Commun. Netw., 2017, doi: 10.1186/s13638-017-0944-x.

[5] M. Maharazu, Z. M. Hanapi, and A. Abdullah, "Call Admission Control for Real-Time and Non-realtime Traffic for Vehicular LTE Downlink Networks," in iCatse International Conference on Mobile and Wireless Technology, 2017, pp. 46-53, doi: 10.1007/978-981-10-5281-1.

[6] M. Al-maitah, O. O. Semenova, A. O. Semenov, P. I. Kulakov, and V. Y. Kucheruk, "A Hybrid Approach to Call Admission Control in 5G Networks," Hindawi Adv. Fuzzy Syst., vol. 2018, 2018.

[7] S. Li, S. Zhao, and S. Zhao, "5G Internet of Things: A Survey," J. Ind. Inf. Integr., 2018, doi: 10.1016/j.jii.2018.01.005.

[8] G. Yan, "Simulation analysis of key technology optimization of 5G mobile communication network based on Internet of Things technology," Int. J. Distrib. Sens. Networks, vol. 15, no. 6, 2019, doi: $10.1177 / 1550147719851454$.

[9] K. H. Slalmi Ahmed, H. C. Saadne Rachid, A. Chehri, and G. Jeon, "Energy Efficiency Proposal for IoT Call Admission Control in 5G Network," in 2019 15th International Conference on Signal-Image Technology \& Internet-Based Systems (SITIS), 2020, pp. 396-403, doi: 10.1109/SITIS.2019.00070.

[10] A. Othman and N. A. Nayan, "Efficient admission control and resource allocation mechanisms for public safety communications over 5G network slice,” Telecommun. Syst., no. 0123456789, 2019, doi: 10.1007/s11235-019-00600-9.

[11] M. Mamman, Z. M. Hanapi, A. Abdullah, and A. Muhammed, "An Adaptive Call Admission Control With Bandwidth Reservation for Downlink LTE Networks,” IEEE Access, vol. 5, pp. 10986-10994, 2017.

(C) 2020 By AIRCC Publishing Corporation. This article is published under the Creative Commons Attribution (CC BY) license. 